\title{
Preferences and Experiences Regarding the Use of the Self-Sampling Device in hrHPV Screening for Cervical Cancer
}

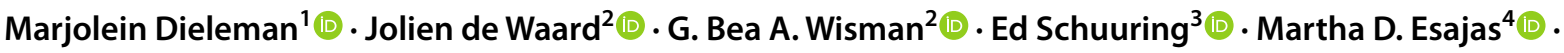 \\ Karin M. Vermeulen ${ }^{1}$ (D) . Geertruida H. de Bock ${ }^{1}$ (D)
}

Accepted: 7 September 2021 / Published online: 24 September 2021

(c) The Author(s) 2021

\begin{abstract}
Background To improve participation in the Dutch cervical cancer screening, a self-sampling device (SSD) was introduced in 2017 into the Dutch population-based screening programme (PBS) for the early detection of cervical cancer. The aim of this study was to gather potential preferences and experiences that might influence a woman's decision to use the SSD in the Dutch PBS.

Methods A scoping review was performed in the PubMed database. Studies that assessed preferences and experiences of women regarding the SSD were included, and preferences and experiences were extracted. In addition, in a qualitative study, the list of potential preferences and experiences specific for the Dutch PBS was extended based on semi-structured interviews with SSD users as well as non-SSD users who recently participated in the PBS, analysed in a structured manner by translating full sentences to key words.

Results Ninety-eight studies were included in the scoping review and 16 interviews were performed. Frequently mentioned reasons for using the SSD, in both the interviews and the literature, were practicality and comfort. Frequently mentioned reasons for not using the SSD were fear of not performing the SSD procedure correctly and doubts on whether the results of the high-risk human papillomavirus (hrHPV) test will be reliable. A new positive experience elicited in the interviews was accessibility. Negative preferences and experiences were not being aware the SSD was an option, and the inconvenience that after an hrHPV-positive test result of the SSD, an additional smear test at the GP is necessary.

Conclusion Several preferences and experiences play a role in the choice whether or not to use the SSD. Based on the currently found preferences and experiences, an app will be developed in order to assess which of these are the most important for women participating in the Dutch population-based cervical screening programme.
\end{abstract}

Marjolein Dieleman and Jolien de Waard contributed equally.

Geertruida H. de Bock

g.h.de.bock@umcg.nl

1 Department of Epidemiology, University of Groningen, University Medical Centre Groningen, Huispostcode FA40, PO BOX 30 001, 9700 RB Groningen, The Netherlands

2 Department of Gynaecologic Oncology, University of Groningen, University Medical Centre Groningen, Groningen, The Netherlands

3 Department of Pathology, University of Groningen, University Medical Centre Groningen, Groningen, The Netherlands

4 Department of Obstetrics and Gynaecology, University of Groningen, University Medical Centre Groningen, Groningen, The Netherlands

\section{Key Points for Decision Makers}

Women's preferences and experiences in the decision to use self-sampling for cervical cancer screening were explored to promote the use of these methods.

A wide variety of these preferences and experiences play a role in this decision, such as the procedure being easy, as represented by a graphic overview (Fig. 3).

This graphic overview can be used in further research to evaluate the most important preferences and experiences. 


\section{Introduction}

Cervical cancer is the fourth most common cancer in women; with an estimated 570,000 new cases and 311,000 deaths in 2018 worldwide [1]. In the Netherlands, the incidence of cervical cancer in a population of around 7 million women at risk, is quite low with 700 women per year diagnosed. However, the number of high-grade lesions is much higher with 5000 diagnoses per year [2]. Nearly all cervical cancers are associated with an infection with high-risk strains of the human papillomavirus (hrHPV) [3]. Most of the HPV infections disappear spontaneously without clinical signs or symptoms. However, if the infection persists, cancer might develop gradually, which can take up to 15-20 years [4]. Cervical cancer is characterized by a well-defined premalignant phase, known as cervical intraepithelial neoplasia (CIN). Identification of clinically relevant high-grade CIN lesions in population-based screening (PBS) programmes and their subsequent treatment have led to a significant reduction in the incidence and mortality of cervical cancer $[5,6]$.

Since 1996, women between the ages of 30 and 60 years in the Netherlands are invited every 5 years to participate in the screening programme [7]. The screening consists of a Pap smear by the general practitioner (GP). As a result of screening, an estimated 700 cases of cervical cancer are prevented annually in the Netherlands [8]. In 2016, however, the participation rate was only $60 \%$ [9]. Since 2017 , the programme has changed to primary hrHPV testing with cytological assessment as triage test. In addition, the possibility to opt-in to receive and use a self-sampling device (SSD) is also available in order to increase the participation in the current PBS. In the first 18 months of the new PBS programme, $8 \%$ of all screened women used an SSD, but overall participation was not increased [10].

To promote the use of the SSD, knowledge on how participation can be improved is essential because a higher participation rate will result in a higher number of early detected high-grade CIN lesions and cervical cancers. Taking the patient's perspective into account is essential in finding explanations to promote the uptake of screening [11]. Earlier studies showed that a woman's choice regarding the screening method for cervical cancer screening depends on multiple factors, for example privacy and embarrassment [12]. However, most of the studies in this field only describe the most common or frequently mentioned experiences and preferences of women. In addition, several studies used closed-ended questionnaires [13-15]. The aim of this study was to construct an extensive and detailed list of possible preferences, experiences and potential participation barriers regarding the use of SSD to identify points upon which the uptake of the screening programme can be improved. Also, a graphic overview of these points will be produced in an online application. Using this application, a small selection of the most important preferences and experiences can be identified in further research which in time can then be used to measure preferences and experiences in different populations. Two consecutive strategies were used. First, a scoping review was performed to identify reported preferences and experiences. Next, semi-structured interviews were held with a small group of women selected by (non) usage of the SSD to elicit additional experiences to complement the existing data from literature.

\section{Methods}

\subsection{Part 1: Scoping Review}

The scoping review, completed on 1 April 2021, involved the MEDLINE database (PubMed), using both MeSH headings and keyword terms in the title or abstract (see Supplementary File 1 in the Electronic Supplementary Material [ESM]). Studies were eligible for inclusion if they met the following criteria: (1) included preferences and experiences regarding a cervicovaginal SSD for cervical screening; and (2) were available as full-text articles in English or Dutch. Studies that did not meet the inclusion criteria or mentioned preferences and experiences of any other type of screening were excluded. In the first step, selection was based on title and abstract screening, and in the second step, selection was based on full-text screening. All publications were reviewed independently by two researchers (MD and JdW). Discrepancies in decisions about inclusion eligibility were resolved through discussion with a third researcher.

\subsubsection{Characteristics of Included Studies}

Characteristics of the selected publications were extracted and summarized by two independent researchers, and added to two descriptive tables. The following data were collected in the first descriptive table: study type, population and setting, type of SSD used or described, primary outcome, study method and the preferences and experiences in favour and against the use of the SSD as mentioned in the conclusion. In the second descriptive table, relevant aspects were assessed based on the Grading of Recommendations Assessment, Development and Evaluation (GRADE) approach, adapted to our study aim [16].

This table includes study type, number of participants, study limitations, characteristics of the studied population in relation to our study population and risk of bias (see Supplementary File 2 and 3 in the ESM). 
Next to both descriptive tables, a complete list of all preferences and experiences mentioned in the full text were extracted per selected publication. Subsequently, all extracted preferences and experiences were combined in one list and those mentioned more than once were merged.

\subsection{Part 2: Semi-Structured Interviews}

According to the Medical Ethical Reviewing Committee (METC) of the University Medical Centre Groningen (UMCG), this study was not subject to the Medical Research Act (WMO). Therefore, no METC approval was needed and an official waiver was received. Prior to the interviews, women were asked to sign a written informed consent form. With this qualitative part of the study, we aimed to gather as many various preferences, experiences and potential barriers as possible. Therefore, three groups of women were included: (1) women who had used the SSD, whose hrHPV result and additional smear test result were positive, and were referred to the hospital for colposcopy; (2) women who had used the SSD, whose hrHPV result was negative and thus were not referred to the GP and (3) women who had not used the SSD, but participated in the PBS through a regular cervical smear collection at the GP for combined hrHPV testing and cytological examination on the same sample. Our aim was to include four women per group. The first group was recruited by gynaecologists at the Department of Gynaecologic Oncology of the UMCG and the Martini Hospital in Groningen, the Netherlands, when visiting the outpatient clinic for colposcopy. The second and third group were recruited through a call on a regional public Facebook group, to which women could respond if they were willing to participate. As the percentage of women using the SSD is low, we reached out to women in the personal or professional network of the research group if the aimed number of four women in the second group was not reached by the intended recruitment methods. For all groups, women were eligible to participate if they were aged 30-60 years old.

The script for the interviews was semi-structured and consisted mainly of open-ended questions about women's preferences and experiences regarding the use of the SSD (see Supplementary File 5 in the ESM). The interview script did not involve any of the results found through the scoping review. The semi-structured interviews were conducted by telephone by two female researchers and three additional students (MD, JdW, VK, EK and EP). Two people were present during each interview, of which one conducted the interview and the other transcribed the content, by writing down the woman's answers. In addition, the interview was recorded by audiotape (with permission of the woman). For women who had no experience with the use of an SSD, a brief explanation of the procedure was provided but no further information on the accuracy or any theoretical background (about hrHPV or cervical cancer) was given. The interviews took about half an hour each, elaborating on every given answer to find as much information as possible. Based on the available literature and our own experience, we would expect data saturation to occur after a total of 12 interviews. The actual number of interviews was considered sufficient if full saturation was reached, meaning that additional participants did not mention any more new preferences and experiences $[17,18]$.

\subsubsection{Analysis of Interviews}

After each interview, its transcription was independently checked for concordance with the content of the audiotape, by a researcher who did not transcribe the actual interview (MD, JdW, VK, EK or EP). Discrepancies were resolved through discussion with at least two researchers involved. The answers given by the participants were structurally analysed in the way proposed by Hsieh and Shannon [19]. The essence of the participants' wording was translated into an umbrella term ('opinion unit'). In case there was no opinion unit available from the ones found in the literature, a new opinion unit was formulated.

\subsection{Part 3: Graphic Display of Results}

A list containing all found preferences and experiences in both the scoping review and the interviews was produced. Dutch results were translated into English. To gain a structured overview of our results, this list was presented in a graphic way called a HealthFan ${ }^{\mathrm{TM}}$ as developed by Château Santé, a tool used in previous research to measure the quality of patients' health status [20]. All individual preferences and experiences, or 'items', were manually divided into main domains that would be based on the themes of the mentioned preferences and experiences. If possible, overlapping terms were combined and further dividing themes (so-called subdomains: a group of preferences and experiences all belonging to one theme within a main domain) were assigned during group sessions with the involved researchers, to make a more structured and compact overview. This overview (HealthFan) was generated in the software of Château Santé and will be available in the future as an online, interactive application.

\section{Results}

\subsection{Part 1: Scoping Review}

The literature search identified 457 publications through database searching using the main search strategy. Of 


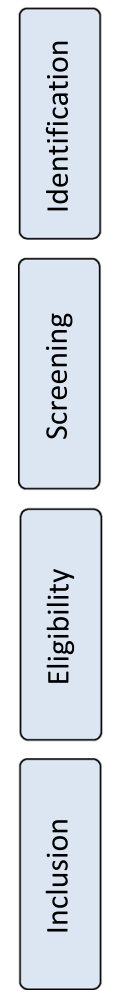

Fig. 1 Flow chart of selected studies

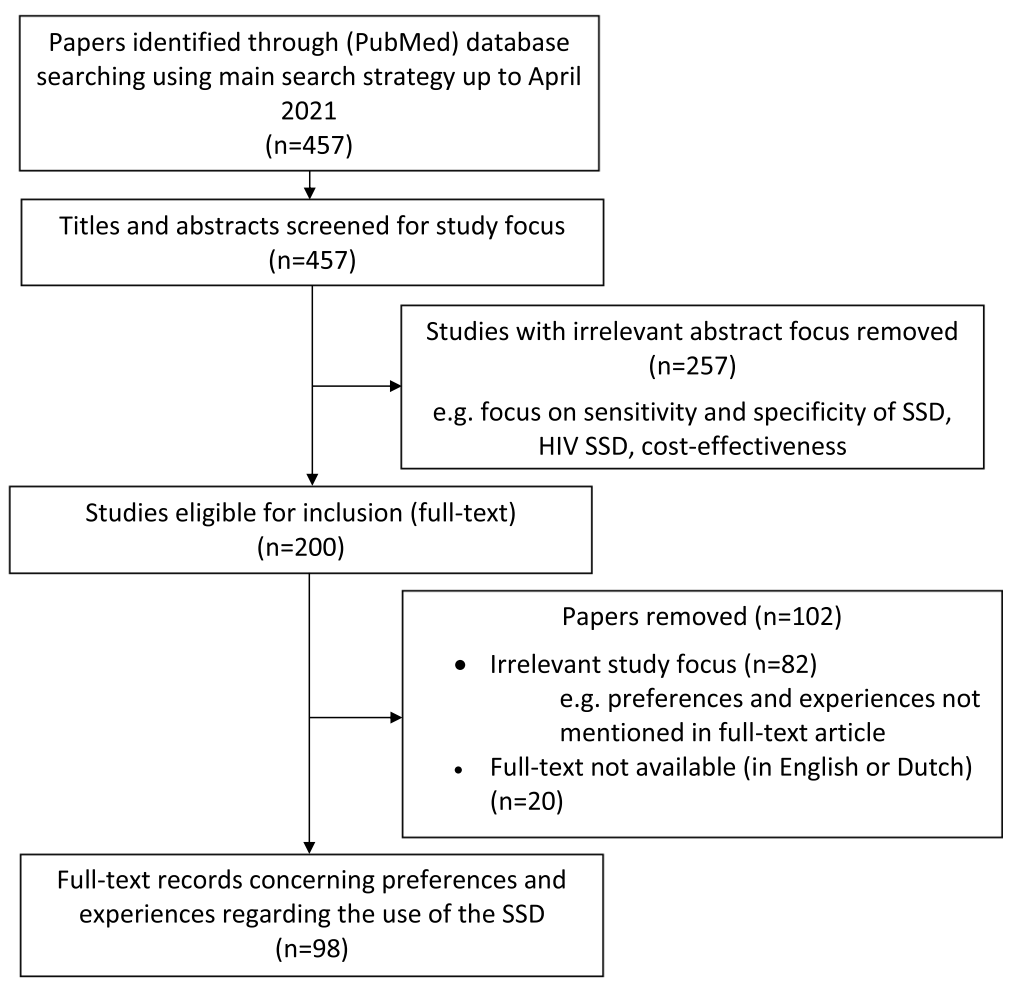

those publications, 257 were removed because of irrelevant abstract focus. Two hundred publications were eligible for inclusion, 102 of those publications were removed based on full-text screening, because they had a non-related study focus or were not available in English or Dutch. Finally, the scoping review included 98 publications (Fig. 1); for references, see Supplementary File 4 in the ESM.

The included studies were survey-based $(N=55)$, qualitative interview studies $(N=21)$, systematic reviews $(N=8)$ and a randomized controlled trial $(\mathrm{RCT})(N=1)$. The remaining studies $(N=13)$ had a mix of the abovementioned designs. The included records assessed the following SSDs: lavage screener, cotton swab, flocked swab, wand device, (cyto)brush and tampon. Studies were performed worldwide and included 12 to 18,202 participants. Detailed characteristics and descriptions can be found in Supplementary File 2 and 3 (see ESM).

Frequently found positive preferences and experiences in literature regarding the use of the SSD were less embarrassment, ease, convenience, comfort and the time-saving aspect. Negative opinion units regarding the use of the SSD included fear of performing the SSD procedure incorrectly, the belief that a physician performs the cervical smear collection better, discomfort and doubts on whether the results of the hrHPV test will be reliable.
All preferences and experiences regarding the use of the SSD found in the scoping review are listed in Supplementary File 6 in the ESM.

\subsection{Part 2: Interviews}

Six women with an hrHPV-positive test followed by positive cytology were recruited for the interviews through the outpatient clinics. Nineteen women responded to the Facebook message. Another three women were recruited through the network of the researchers, as we did not succeed in recruiting the intended number of women by the first two methods. Interviews were conducted with 16 out of 28 women that were willing to participate (Fig. 2). The age of the participants ranged from 30 to 51 years, with a mean age of 37.6 years and the educational level ranged from vocational to academic education. Main preferences and experiences mentioned by our participants and not found through our scoping review were as follows: using the SSD is approachable, the SSD is a friendly device and that it is easy to drop the SSD in the mailbox. For example, participant 1 (aged 38 years, referred to the hospital after an HPV+ result of the SSD) mentioned "It [the SSD] is approachable. I think people would rather use the SSD". On the other hand, women also mentioned the following possible barriers 


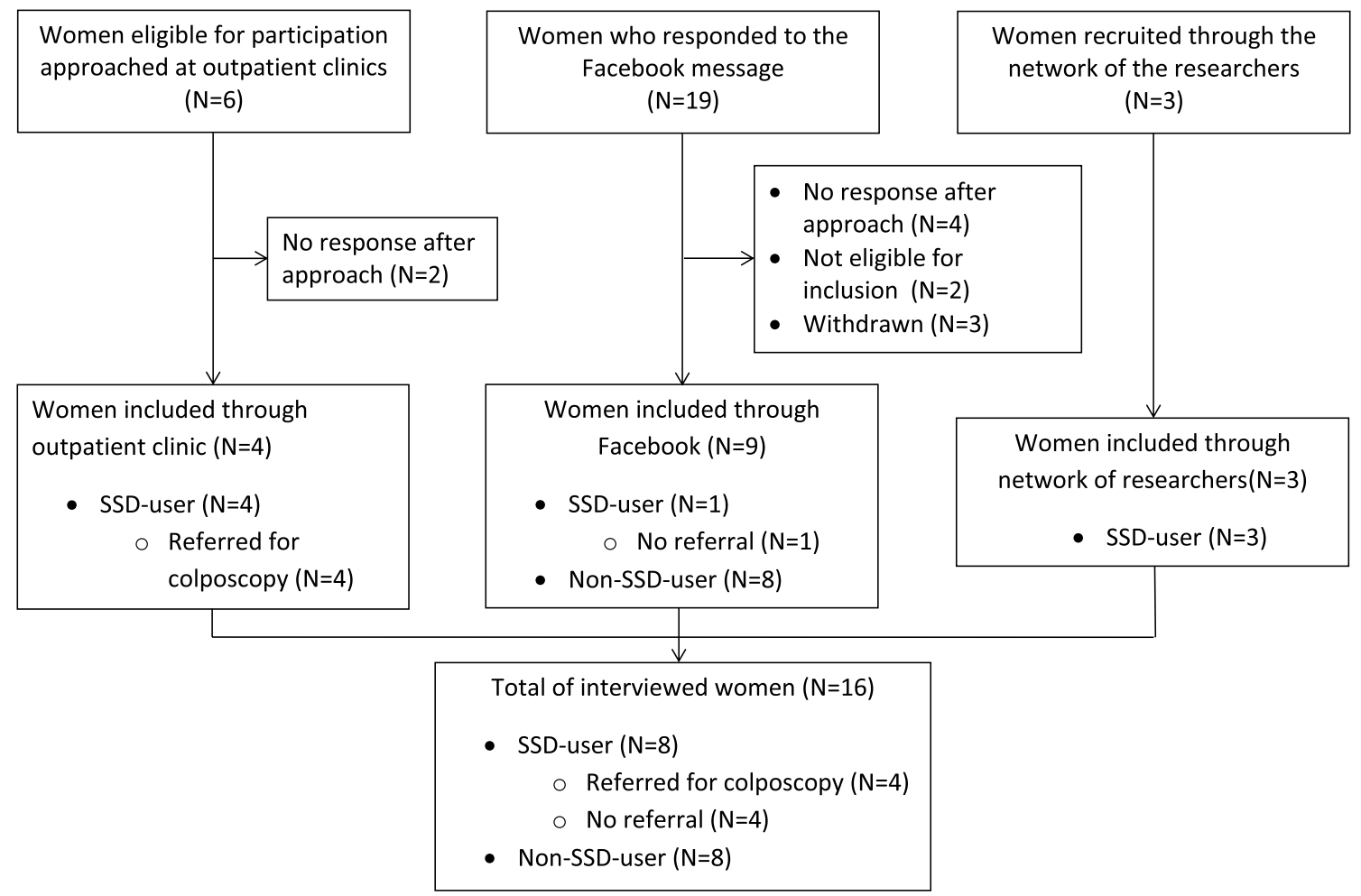

Fig. 2 Flow chart of included women

for using the SSD: the option for the SSD is not directly mentioned, it is inconvenient because of the need to have an additional cervical smear collected by the GP when the SSD result is hrHPV-positive and difficulties (e.g. requesting the SSD is difficult) (see Supplementary File 7 in the ESM). For example, participant 4 (aged 32 years, referred to the hospital after an HPV+ result of the SSD) mentioned "It [the result of the SSD] was not good, so you have to go for a smear test anyway, the SSD almost feels like an extra step". After interviewing four to five women per category, no new preferences and experiences were found. Based on the saturation criterion, this number of interviews was thus considered sufficient.

\subsection{Part 3: Graphic Display of Found Preferences and Experiences Regarding Self-Sampling Device (SSD) Use}

After performing the scoping review and the interviews, results were combined in one list. In the process of generating the HealthFan, all items had a content related to one of the following three domains: physical, psychosocial and practical (Fig. 3 and Supplementary File 8 of the ESM), and were divided accordingly.

The physical domain involves aspects associated with the physical experiences encountered in performing the SSD procedure. It consists of one subdomain, called 'body', and five items, including 'fear of injury', 'pain level' and 'positioning'.

The psychosocial domain consists of nine subdomains and 19 items. This domain is filled with psychological or social aspects regarding the SSD. The largest subdomain is called 'feelings', containing items such as 'experienced safety', 'embarrassment' and 'nervousness'.

The practical domain, involving practical aspects of the $\mathrm{SSD}$, is the largest domain and consists of ten subdomains and twenty items. The subdomains with the most items are 'ease of use', 'request procedure and instructions' and 'time saving'. Items in the subdomain 'ease of use' include 'procedure', 'accessibility' and 'transportation'.

Figure 3 is a static depiction of the HealthFan. The HealthFan will be accessible as an online, interactive application, in which individual items can be selected. Subsequently, selected items can be ranked from most to least important and information can be gathered on which items are selected most frequently. Based on this, we can determine which preferences and experiences are most important. 


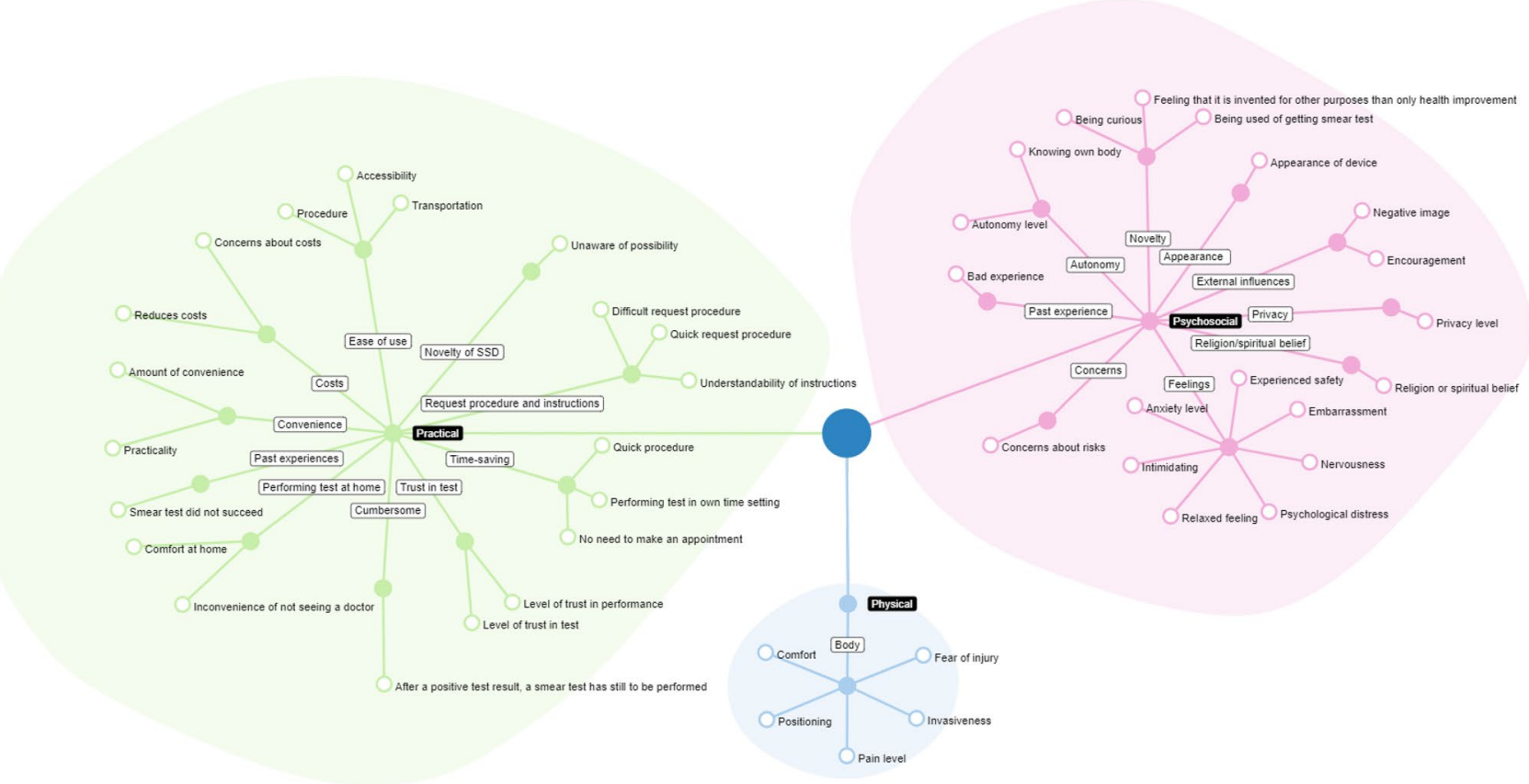

Fig. 3 HealthFan

\section{Discussion}

In this study, a scoping review was performed as well as semi-structured interviews, which were performed to yield any extra preferences and experiences specifically for the Dutch PBS. Several factors played a role in women's considerations regarding the use of the SSD. Ease, convenience and the time-saving aspects of the SSD were stated as positive aspects in this and other studies [e.g. 12, 20-22], whereas negative preferences and experiences included the fear of performing the SSD procedure incorrectly and doubts on whether the results of the hrHPV test will be reliable. The interview results were in alignment with the results from the scoping review. However, our participants additionally mentioned a few aspects that were specific for the Dutch PBS, such as the need for having an additional cervical smear collected by the GP when the SSD result is hrHPV-positive and that the procedure to request the device was difficult. These only represent a fraction of the many preferences and experiences we found, the rest of which are uniquely displayed in our HealthFan (Fig. 3).

The aim of this study was to obtain a broad view of possible preferences and experiences. For that, we first did a systematic search of the literature following a strict method of selecting and reading studies, including the assessment of risk of bias. However, we did not perform a quality assessment of the derived information, as the quality of the reviewed studies is of less relevance for the aim of our scoping review, as this aim was to collect the broadest possible range of preferences and experiences.

Most preferences and experiences found through the interviews were covered by the findings of our scoping review. The interviews, however, yielded some additional preferences and experiences of value. An explanation is that the Dutch PBS is organised differently than screening programmes in other countries and that the studied population is different. This was also reported by Oranratanaphan et al. [24], illustrating that differences in acceptability of the SSD is influenced by regional culture and behaviour. Another explanation is our use of semi-structured interviews with open-ended questions. This enabled women to elaborate on their opinion and mention more individual, less typical preferences and experiences, whereas in many other studies this possibility was not offered because mainly closedended questionnaires were used (Supplementary Files 2, 3, see ESM). In addition, most other studies evaluated whether self-sampling is an acceptable self-collection method among women [22, 24, 28], whereas we aimed to find an extensive number of preferences and experiences regarding the use of the SSD.

To our knowledge, this is the first study investigating preferences and experiences regarding the SSD by combining a scoping review with qualitative in-depth interviews. Next to this, a lot of effort was put in preserving the linguistic nuances from our interview results. In contrast to other studies in which preferences and experiences were often fitted into larger umbrella themes (e.g. 'preferring a smear test'), 
we set out the different underlying reasons that were mentioned in our interviews ('preferring a smear test' because 'a health professional can perform more thorough inspection'), leading to a broader insight into the possible factors. This interview method is a main strength of our study, along with the unique combination of study designs.

A limitation of this study is that all our participants were already actively involved in the national screening programme, as they all responded to the invitations by either having a cervical smear taken by the GP or using the SSD. With our selection approach we probably did not reach women not participating in the screening programme. This might have introduced a selection bias. However, our scoping review did include studies focussing on 'hard-to-reach' women and non-attendees to the screening programme [15, $21,23,29]$. Secondly, we only assessed studies published in the PubMed database, which can be considered as a limitation as we realize our search might not have been ultimately complete. However, as PubMed is the most commonly used database for medical publications, we are confident we found the most relevant preferences and experiences to reach our aim, to gather potential preferences and experiences that might influence a woman's decision to use the SSD in the Dutch PBS, with the perspective of developing an instrument suitable for our population. Thirdly, we did not perform a quantitative quality assessment on the included studies. The main reason for that is that the criteria posed for quality of quantitative studies were not applicable to our study, as we were qualitatively searching for as many mentioned and described preferences and experiences as possible. Therefore, a qualitative interview study with only few participants could be more valuable to our research than a large systematic review, while this would be graded low in quality according to the GRADE criteria. Lastly, 16 interviews might be considered a small sample size. However, this concerns a qualitative study with the goal being to find as many preferences as possible, instead of providing a quantitative assessment of frequency of occurrence of the various preferences and experiences. As we included numerous studies with experiences and preferences and a saturation of new items was reached during the interviews, we are convinced that we covered the wide range of potential preferences and experiences. Performing a larger number of interviews would not have been of additional value, since this would most likely not have elicited any addition preferences and experiences.

To provide optimal healthcare, it is crucial to also take the patient's perspective into account. In this way, engagement of patients in their own self-care, and thereby commitment, might be improved [30]. This seems especially important in preventive medicine, in which patients are expected to participate without clear benefit (which is different from, for example, making use of the healthcare system when having explicit complaints) and negative experiences or practical objections may even more easily raise the threshold to participate. Evaluating the acceptability of the SSD, which was done in this study by making use of women's preferences and experiences, can help to improve the patient experience of the general cervical cancer screening programme and thereby potentially enhancing the participation rate. This is obviously very important in early detection and treatment of (pre)cancerous cervical lesions, thereby lowering the burden of disease in the general population.

The present qualitative study resulted in a patient-centred instrument to measure preferences regarding screening, namely our structured graphic overview (Fig. 3). This is an important first step in taking the patient's perspective into account in the process of optimizing cervical cancer screening programmes and such tools could be used in further research to explore important factors. Our extensive list of preferences and experiences (Supplementary File 6 and 7, see ESM) could be used for setting up a similar, countryspecific tool. Specifically regarding the Dutch PBS, the next step will be to ask patients in the Dutch population to select the most important preferences and experiences. The 9-12 most important aspects will be incorporated in an instrument (an app for mobile devices) to generate empirical results regarding the extent of women's preferences and experiences measured on a four-point scale. In this way, we aim to gain insights into how Dutch participation rates could be improved.

\section{Conclusion}

In this study, we found several preferences and experiences to play a role in the choice of whether or not to use the SSD, such as ease and convenience, but also the fear of performing the SSD procedure incorrectly. Based on the currently found preferences and experiences, an extensive graphic overview was generated, which will be used to assess the most important preferences and experiences for women participating in the Dutch population-based cervical screening programme.

Supplementary Information The online version contains supplementary material available at https://doi.org/10.1007/s40271-021-00550-y.

Acknowledgements This study was funded by the Netherlands Organisation for Health Research and Development, ZonMw. The authors wish to thank the women who participated in the interviews. We also thank Eva Peters, Eline Klaas and Veerle Kisjes for their assistance during the interviews and the scoping review. Also, we would like to thank Dr. Paul Krabbe (Dept. Epidemiology, UMC Groningen) for sharing his expertise in the use of the HealthFan software. 


\section{Declarations}

Funding This study was funded by the Netherlands Organisation for Health Research and Development, ZonMw.

Conflict of interest The authors have no conflicts of interest.

Availability of data and material All data are available on request.

Code availability Not applicable.

Author contributions Conception and design: MD, JdW, GBAW, ES, KMV, MDE, GHdB. Development of methodology: MD, JdW, GHdB, GBAW, KMV. Acquisition of data (performing scoping review and interviews, finding and including patients): MD, JdW, MDE. Interpretation and processing of data: $\mathrm{MD}, \mathrm{JdW}$. Assembly and writing of manuscript: MD, JdW, GBAW, ES, MDE, KMV, GHdB.

Ethics approval This study was not subject to the Medical Research Act (WMO). An official waiver was received.

Consent to participate Informed consent was obtained from all individual participants included in the study.

Consent for publication All authors have reviewed and approved the manuscript and consent to its publication.

Open Access This article is licensed under a Creative Commons Attribution-NonCommercial 4.0 International License, which permits any non-commercial use, sharing, adaptation, distribution and reproduction in any medium or format, as long as you give appropriate credit to the original author(s) and the source, provide a link to the Creative Commons licence, and indicate if changes were made. The images or other third party material in this article are included in the article's Creative Commons licence, unless indicated otherwise in a credit line to the material. If material is not included in the article's Creative Commons licence and your intended use is not permitted by statutory regulation or exceeds the permitted use, you will need to obtain permission directly from the copyright holder. To view a copy of this licence, visit http://creativecommons.org/licenses/by-nc/4.0/.

\section{References}

1. Bray F, Ferlay J, Soerjomataram I, Siegel RL, Torre LA, Jemal A. Global cancer statistics 2018: GLOBOCAN estimates of incidence and mortality worldwide for 36 cancers in 185 countries. CA Cancer J Clin. 2018;68:394-424.

2. Integraal Kankercentrum Nederland. Monitor bevolkingsonderzoek baarmoederhalskanker 2018. 2019. https://www.rivm.nl/ documenten/monitor-bevolkingsonderzoek-baarmoederhalsk anker-2018

3. Nelson EJ, Maynard BR, Loux T, Fatla J, Gordon R, Arnold LD. The acceptability of self-sampled screening for HPV DNA: a systematic review and meta-analysis. Sex Transm Infect. 2017;93:56-61.

4. WHO. Human papillomavirus (HPV) and cervical cancer. 2019. https://www.who.int/news-room/fact-sheets/detail/human-papil lomavirus-(hpv)-and-cervical-cancer. Cited 30 Mar 2020.

5. Arbyn M, Raifu AO, Weiderpass E, Bray F, Anttila A. Trends of cervical cancer mortality in the member states of the European Union. Eur J Cancer. 2009;45:2640-8. https://doi.org/10.1016/j. ejca.2009.07.018
6. Peto PJ, Gilham PC, Fletcher O, Matthews FE. The cervical cancer epidemic that screening has prevented in the UK. Lancet. 2004;364:249-56.

7. van der Veen N. Framework for the execution of cervical cancer population screening [in Dutch]. Bilthoven: Rijksinstituut voor Volksgezondheid en Milieu (RIVM); 2017.

8. Naber SK, Matthijsse SM, Jansen EEL, de Kok IMCM, de Koning HJ, van Ballegooijen M. Effecten en kosten van het vernieuwde bevolkingsonderzoek naar baarmoederhalskanker in Nederland naar aanleiding van recente ontwikkelingen. 2016. https://www. rivm.nl/documenten/kosteneffectiviteitsanalyse-bevolkingsonder zoek-baarmoederhalskanker

9. PALGA, Erasmus MC. Bevolkingsonderzoek baarmoederhalskanker monitor 2016. 2016. https://www.rivm.nl/documenten/ landelijke-evaluatie-van-bevolkingsonderzoek-baarmoederhalsk anker-leba-tm-2016

10. Aitken CA, Van Agt HME, Siebers AG, Van Kemenade FJ, Niesters HGM, Melchers WJG, et al. Introduction of primary screening using high-risk HPV DNA detection in the Dutch cervical cancer screening programme: a population-based cohort study. BMC Med. 2019;17:1-14.

11. Ragas DM, Nonzee NJ, Tom LS, Phisuthikul AM, Luu TH, Dong XQ, et al. What women want: patient recommendations for improving access to breast and cervical cancer screening and follow-up. Women's Heal Issues. 2014;24:511-8.

12. Bosgraaf RP, Ketelaars PJW, Verhoef VMJ, Massuger LFAG, Meijer CJLM, Melchers WJG, et al. Reasons for non-attendance to cervical screening and preferences for HPV self-sampling in Dutch women. Prev Med (Baltim). 2014;64:108-13. https://doi. org/10.1016/j.ypmed.2014.04.011.

13. Bansil P, Wittet S, Lim JL, Winkler JL, Paul P, Jeronimo J. Acceptability of self-collection sampling for HPV-DNA testing in low-resource settings: a mixed methods approach. BMC Public Health. 2014;14:1-9.

14. Allende G, Surriabre P, Cáceres L, Bellot D, Ovando N, Torrico A, et al. Evaluation of the self-sampling for cervical cancer screening in Bolivia. BMC Public Health. 2019;19:1-9.

15. Polman NJ, de Haan Y, Veldhuijzen NJ, Heideman DAM, de Vet HCW, Meijer CJLM, et al. Experience with HPV self-sampling and clinician-based sampling in women attending routine cervical screening in the Netherlands. Prev Med (Baltim). 2019;125:5-11. https://doi.org/10.1016/j.ypmed.2019.04.025.

16. Guyatt G, Oxman AD, Akl EA, Kunz R, Vist G, Brozek J, et al. GRADE guidelines: 1 Introduction-GRADE evidence profiles and summary of findings tables. J Clin Epidemiol. 2011;64:383-94.

17. Moser A, Korstjens I. Series: practical guidance to qualitative research. Part 3: sampling, data collection and analysis. Eur J Gen Pract. 2018;24:9-18. https://doi.org/10.1080/13814788.2017. 1375091.

18. Crouch M, McKenzie H. The logic of small samples in interviewbased qualitative research. Soc Sci Inf. 2006;45:483-99.

19. Hsieh HF, Shannon SE. Three approaches to qualitative content analysis. Qual Health Res. 2005;15:1277-88.

20. Château Santé. Château Santé [Internet]. 2019. https://www.chate au-sante.com/. Cited 16 Apr 2019

21. Andersson S, Belkić K, Mints M, Östensson E. Acceptance of self-sampling among long-term cervical screening non-attenders with HPV-positive results: promising opportunity for specific cancer education. J Cancer Educ. 2021;36:126-33.

22. Braz NSDF, Lorenzi NPC, Sorpreso ICE, de Aguiar LM, Baracat EC, Soares-Júnior JM. The acceptability of vaginal smear selfcollection for screening for cervical cancer: a systematic review. Clinics. 2017;72:183-7. 
23. Virtanen A, Nieminen P, Niironen M, Luostarinen T, Anttila A. Self-sampling experiences among non-attendees to cervical screening. Gynecol Oncol. 2014;135:487-94.

24. Oranratanaphan S, Termrungruanglert W, Khemapech N. Acceptability of self-sampling HPV testing among thai women for cervical cancer screening. Asian Pac J Cancer Prev. 2014;15:7437-41.

25. Quincy BL, Turbow DJ, Dabinett LN. Acceptability of self-collected human papillomavirus specimens as a primary screen for cervical cancer. J Obstet Gynaecol (Lahore). 2012;32:87-91.

26. Hanley SJB, Fujita H, Yokoyama S, Kunisawa S, Tamakoshi A, Dong P, et al. HPV self-sampling in Japanese women: a feasibility study in a population with limited experience of tampon use. J Med Screen. 2016;23:164-70.
27. Lorenzi NPC, Termini L, Longatto Filho A, Tacla M, de Aguiar LM, Beldi MC, et al. Age-related acceptability of vaginal selfsampling in cervical cancer screening at two university hospitals: a pilot cross-sectional study. BMC Public Health. 2019;19:963.

28. Tisci SE, Shen YH, Fife D, Huang J, Goycoolea J, Ma CP, et al. Patient acceptance of self-sampling for human papillomavirus in rural China. J Low Genit Tract Dis. 2003;7:107-16.

29. Kilfoyle KA, Des Marais AC, Ngo MA, Romocki L, Richman AR, Barclay L, et al. Preference for human papillomavirus selfcollection and papanicolau: survey of underscreened women in North Carolina. J Low Genit Tract Dis. 2018;22:302-10.

30. Black N. Patient reported outcome measures could help transform healthcare. BMJ. 2013;346:1-5. 